\title{
Dye-doped spheres with plasmonic semi-shells: Lasing modes and scattering at realistic gain levels
}

\author{
Nikita Arnold*, Boyang Ding§, Calin Hrelescu and Thomas A. Klar
}

\section{Full Research Paper}

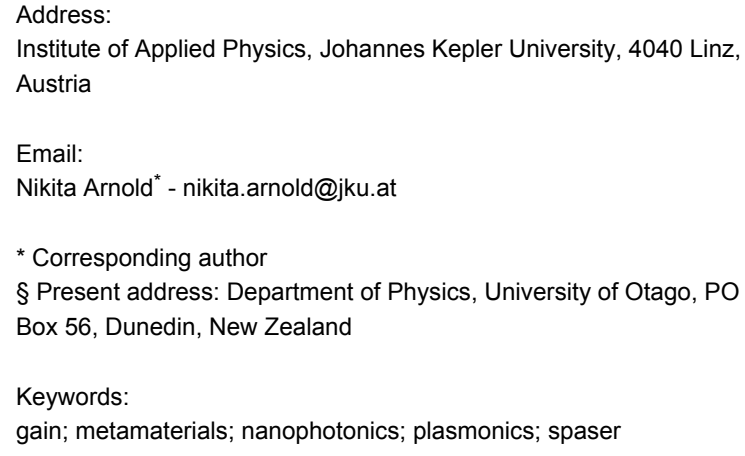

Beilstein J. Nanotechnol. 2013, 4, 974-987. doi:10.3762/bjnano.4.110

Received: 28 August 2013

Accepted: 09 December 2013

Published: 30 December 2013

This article is part of the Thematic Series "Optical near-fields \& nearfield optics".

Guest Editors: A. J. Meixner and P. Leiderer

(C) 2013 Arnold et al; licensee Beilstein-Institut. License and terms: see end of document.

\begin{abstract}
We numerically simulate the compensation of absorption, the near-field enhancement as well as the differential far-field scattering cross section for dye-doped polystyrene spheres (radius $195 \mathrm{~nm}$ ), which are half-covered by a silver layer of 10-40 nm thickness. Such silver capped spheres are interesting candidates for nanoplasmonic lasers, so-called spasers. We find that spasing requires gain levels less than 3.7 times higher than those in commercially available dye-doped spheres. However, commercially available concentrations are already apt to achieve negative absorption, and to narrow and enhance scattering by higher order modes. Narrowing of the plasmonic modes by gain also makes visible higher order modes, which are normally obscured by the broad spectral features of the lower order modes. We further show that the angular distribution of the far-field scattering of the spasing modes is by no means dipole-like and is very sensitive to the geometry of the structure.
\end{abstract}

\section{Introduction}

Noble metal nanoparticles are of current interest both in fundamental and applied science because of their localized plasmonic resonances (LPR) in the visible and near infrared range of the optical spectrum. The simplest nanoparticle geometry is spherical, but the spectral position of the nanoparticle plasmon of a solid sphere can only be tuned by increasing its radius (given a specific refractive index of the embedding medium), which leads to a redshift with increasing radius [1]. The price one has to pay for this size-based wavelength-tuning is, however, a substantial spectral broadening due to radiative damping. An alternative way to tune the LPR spectrally is to change the shape of the nanoparticle. First, one can relax the radial homogeneity of the nanoparticle and turn from solid nanoparticles to noble metal nanoshells [2,3]. Second, one can also relax the angular symmetry and turn from nanoshells with spherical symmetry to semi-shells, sometimes also called nanocaps or nano-cups. Such semi-shells can be produced either via the evaporation of noble metals on top of dielectric spheres 
[4-6], via the electrochemical deposition through a self-assembled template of dielectric spheres [7], via the attachment of seed particles to dielectric spheres that are partially embedded in a polymer matrix and a subsequent electroless plating [8], or via opening holes in originally closed shells via e-beam sputtering [9] or ion beam milling [10].

The semi-shells show a rich spectrum of localized plasmon resonance modes that was investigated analytically and numerically [11-13]. It became common to label the different modes according to the symmetries of the modes of the spherically symmetric closed metallic shells. The semi-shell modes can be derived from the closed-shell modes by slowly opening the closed shell. Labeling is hence carried out by using the multipolar and azimuthal numbers $(l, m)[7,11]$. While in a spherically symmetric shell, each multipolar mode is $(2 l+1)$ times degenerate, the symmetry breaking that is introduced by opening a hole in the shell not only lifts this degeneracy, but also introduces a further mode splitting via the coupling of the shell plasmons with the rim plasmon modes of the circular opening [11]. The excitability of the localized plasmon modes depends on the polar and azimuthal angles of the direction of illumination and on the orientation of the electric field vector. In a recent experimental work, some of the most prominent eigenmodes of metallic voids have been imaged using a nearfield microscope [14]. A further prominent feature of semishells with broken symmetry (compared to angularly symmetric shells) is their capability to scatter light preferentially into certain directions $[15,16]$. Recently, we have shown that localized plasmonic modes of the semi-shells are apt to direct the fluorescence from the dyes contained in their dielectric cores into the forward direction [17]. Plasmonic semi-shells or nanovoids have also been used for important applications such as biosensing [18], plasmon-enhanced solar cells [19,20], or as substrates for surface-enhanced Raman scattering [21,22] and coherent anti-Stokes Raman scattering [23].

A severe problem for all plasmonic applications is the damping of plasmons due to Ohmic losses in the metal and due to radiative losses. However, a solution for this dilemma is possible because plasmons are Bosons and can hence be emitted via stimulated emission [24]. This might be used to minimize losses in metamaterials $[25,26]$ and it has also been proposed for the compensation of losses in plasmonic shells [27,28]. Losscompensation in the case of solid gold nanospheres embedded in a gain medium has been experimentally verified by Noginov et al. [29] and more recently confirmed by Strangi et al. [30]. The cancellation of the losses in hybrid materials that comprise resonant nanoparticle plasmons and gain materials finally leads to a self-sustaining laser-like generator called the spaser [3136]. In this contribution, we find that there is a finite range of gain values in which the absorption is overcompensated within a certain wavelength region, but the spaser does not self-start yet. The existence of such a finite range has been debated before [37-41].

A true nano-spaser based on localized plasmons should be subwavelength confined in all three dimensions (3D). Experimentalists approached such 3D nano-spasers within the last few years [42-48]. The smallest nano-spaser so far has been claimed by a team around Noginov, Shalaev and Wiesner [49] who reported that gold nanoparticles with a diameter of $14 \mathrm{~nm}$, covered by a silica shell of $15 \mathrm{~nm}$ thickness, doped with the dye OG-488 show laser emission. The nanostructures reported in this experimental study were, however, fully spherically symmetric. Very recently, it has been proposed that symmetry breaking might have advantages because a low gain threshold is required [50] and the coherent emission becomes directional [51]. In both theoretical papers, a geometry was assumed, in which a dye-doped dielectric sphere was covered by a semishell of noble metals.

The authors of [50,51] assumed metal-capped and dye-doped spheres comprising a spectrally flat, dispersion-less gain. This does not reflect realistic dye molecules, which provide gain only within a limited spectral range. However, it is a useful approach to search for the spectral mode that will lase most easily. In this work, we would like to take the complementary approach by starting with two given Lorentzian lines for the molecular absorption and the Stokes-shifted emission of a given realistic dye molecule that fluoresces in the visible region. We tune specific plasmonic resonances into the emission maximum of the dye molecules by adjusting the thickness of the silver caps on the dye-doped spheres. In particular, we assume a doping concentration, a size of the spheres and the type of dye molecules as given by the commercially available polystyrene spheres "Firefli* Fluorescent Green" from Thermo-Scientific, Waltham, MA, USA, which we have used in an experimental study on the spectral and directional Purcell effect [17]. With this self-restriction to the parameters of commercially available dye-doped spheres, we make a realistic estimate of how far away one is from nanoparticle spasing (we find: a factor of 3.7 in necessary gain, only) and which other effects, such as a spectral narrowing of the scattering cross section or a change in absorption should already be observable in the case of the available gain. We find that narrowing of the Mie scattering spectrum and small signal gain could become detectable.

\section{Numerical modeling}

Figure 1 depicts the geometry of the silver capped spheres and the orientation of the incoming electromagnetic wave. A dyedoped polystyrene (PS) sphere of $390 \mathrm{~nm}$ diameter is capped 
with a semi-shell of silver. The coordinate system is fixed such that the z-axis defines the axis of rotational symmetry of the semi-shell and points inwards into the metallic cap. The direction of the $\boldsymbol{k}$-vector of the linearly polarized plane wave is given by two spherical angles $(\theta, \varphi)$ in which $\theta$ denotes the polar angle between the $\boldsymbol{k}$-vector and the $\mathrm{z}$ axis, and $\varphi$ denotes the azimuthal angle in the $\mathrm{x}-\mathrm{y}$ plane. The electric field always remains in the $\mathrm{x}-\mathrm{z}$ plane. In particular, the $(0,0)$ direction denotes an illumination from the open, uncovered side of the PS sphere (as shown in Figure 1), while $(180,0)$ denotes an illumination from the silver-capped side and $(90,0)$ denotes an illumination from the side, in which the electric field is parallel to the z-axis such that axial plasmonic modes can be excited. The thickness of the silver shell and the concentration of the dye molecules are varied throughout the paper.

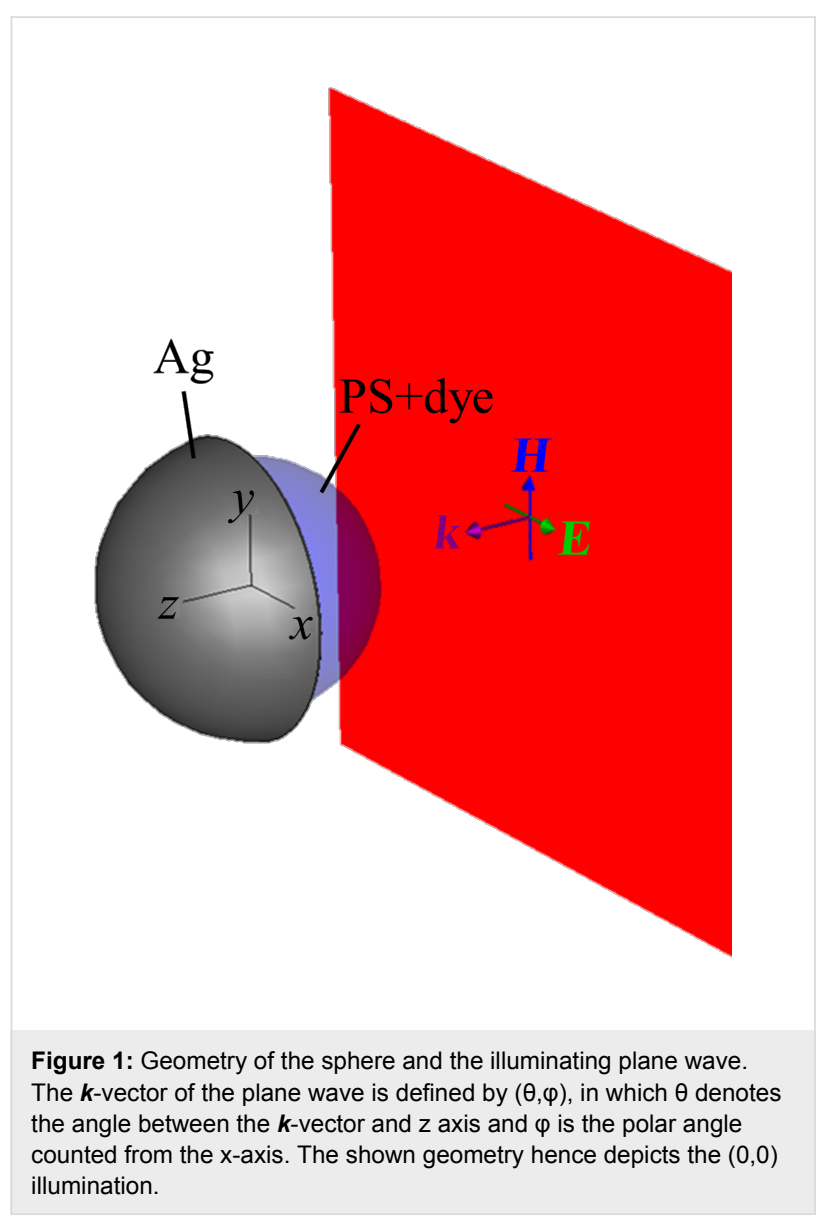

Realistic modeling of 3D plasmonic structures with gain in the visible-light range is non-trivial and requires certain care. As mentioned before, semi-shell structures possess many multipolar eigenmodes that are located closely together in frequency space [11], and many of the modes are easily overlooked when modeling is carried out without gain, because they are strongly damped because of the dispersion of the metal. If, however, damping is compensated by gain, the modes might become ultra-sharp and can still be overlooked if they are narrower than the frequency step used in simulations. The situation is somewhat similar to the scattering by weakly dissipating plasmonic spheres, in which very narrow higher order resonances can dominate [52]. These effects are even more pronounced for the anisotropic dielectric permittivity with transverse or longitudinal gain [53]. Computational details are discussed in the section "Numerical" at the end of the paper.

The dielectric constant of silver was taken from Johnson and Christy [54]. The dielectric constant of the gain material was described by a double Lorentzian function:

$$
\begin{aligned}
\varepsilon_{\text {Sphere }} & =\varepsilon_{\mathrm{PS}}+\mathrm{A} \cdot \Delta \varepsilon_{\mathrm{Dye}} \frac{\omega_{\mathrm{abs}}^{2}}{\omega_{\mathrm{abs}}^{2}-\omega^{2}-i \omega \gamma} \\
& -\mathrm{E} \cdot \Delta \varepsilon_{\mathrm{Dye}} \frac{\omega_{\mathrm{em}}^{2}}{\omega_{\mathrm{em}}^{2}-\omega^{2}-i \omega \gamma}
\end{aligned}
$$

The coefficient $\Delta \varepsilon_{\text {Dye }}$ describes the strength of the transition (for the time being the same strength of absorption and emission is assumed [55]), $\omega_{\mathrm{abs}}=4.02 \cdot 10^{15} \mathrm{~s}^{-1}(468 \mathrm{~nm})$ and $\omega_{\mathrm{em}}=3.67 \cdot 10^{15} \mathrm{~s}^{-1}(513 \mathrm{~nm}), \gamma=5.0 \cdot 10^{14} \mathrm{~s}^{-1}\left(\Delta \lambda_{\mathrm{FWHM}} \approx\right.$ $60 \mathrm{~nm}$ ), and we assume a purely real $\varepsilon_{\mathrm{PS}}=2.6$ for polystyrene. The coefficient A is set to " 1 " and the coefficient $\mathrm{E}$ is set to " 0 " if we assume a purely absorbing medium (all dye molecules in the ground state) and $\mathrm{A}=0, \mathrm{E}=1$ in case of a fully inverted medium. Coefficients between 0 and 1 are used to simulate partial inversions, in which $\mathrm{A}+\mathrm{E}=1$ holds.

Unsaturated Lorentzian gain does not allow for simulations very close to the lasing threshold (leading to unphysical infinite scattering cross sections and a diverging gain $[36,56])$, nor does it have much of a physical meaning beyond the threshold in CW operation. However, a classical electromagnetic calculation with a Lorentzian gain is fully legitimate as long as the lasing threshold is approached from below [36]. Here, we define the extinction cross section as $\sigma_{\mathrm{ex}}=\sigma_{\mathrm{sc}}+\sigma_{\mathrm{ab}}$, irrespectively of the sign of $\sigma_{\mathrm{ab}}$.

To get realistic simulation parameters, we determined the chromophore density in dye-doped polystyrene (PS) spheres, which are commercially available from Thermo-Scientific (Waltham, MA, USA). Those spheres are doped with "Firefli* Fluorescent Green" dye with an absorption peak near $468 \mathrm{~nm}$ and an emission peak at $513 \mathrm{~nm}$. The concentration of the dye molecules inside the PS spheres was derived by taking the optical density (OD) of a solution of dye-doped spheres and subtracting the scattering component of the OD. The concentration of the 
spheres was quantified by measuring the scattering strength at a wavelength far from the absorption of the dyes. From this, we estimated an absorption constant in the range of $2300 \mathrm{~cm}^{-1}$, which translates into a transition strength of $\Delta \varepsilon_{\text {Dye }}=0.004$. In the simulations, we often considered cases of pure absorption or pure emission, which correspond to the cases in which all the dye molecules are in the absorbing ground state or all dye molecules are in the emitting state respectively (full inversion, which is achievable in a four level system). We also considered a 50/50 combination of two Lorentzian lines, which corresponds to half of the chromophores being excited $(A=E=1 / 2$ in Equation 1). For the dye concentration of these commercially available PS spheres, we do not observe any spasing, yet. Hence, we also considered substantially higher dye concentrations, i.e., we applied $\Delta \varepsilon_{\text {Dye }}>0.004$. Primarily for didactical reasons we also went to dye concentrations beyond the lasing threshold. As a reference, the peak of the imaginary part of the dielectric constant $\varepsilon_{\max }^{\prime \prime}$ is related to $\Delta \varepsilon_{\text {Dye }}$ at the central emission wavelength via the equation $\varepsilon_{\max }^{\prime \prime}=\Delta \varepsilon_{\text {Dye }} \cdot \omega_{\mathrm{em}} / \gamma=$ $7.34 \Delta \varepsilon_{\text {Dye }}$.

\section{Results and Discussion Matching the plasmonic resonance to the gain spectrum}

Before we include the gain material in the calculations, we tune the plasmonic resonance of the semi-shells to overlap with the emission band of the fluorophores by adjusting the thickness $h$ of the silver caps on the undoped PS spheres of $390 \mathrm{~nm}$ diameter. Figure 2 shows the extinction, scattering, and absorption spectra in panels a,b,c, respectively, for thicknesses varying from $h=10$ to $40 \mathrm{~nm}$ as indicated by the color coding. The linearly polarized, electromagnetic plane wave impinges the structure from the "open sphere side", i.e., in the $(0,0)$ direction as depicted in Figure 1. In Figure $2 \mathrm{~d}$ all three cross sections are compared for the specific sample with $h=20 \mathrm{~nm}$, showing that the total extinction spectrum is dominated by the scattering. However, the most prominent resonances are seen in the absorption spectra. On the black curve $(h=10 \mathrm{~nm})$ in Figure 2c, one clearly sees several plasmonic maxima, for which the maxima become sharper with decreasing wavelength. When the thickness of the semi-shells is decreased, the series of absorp-

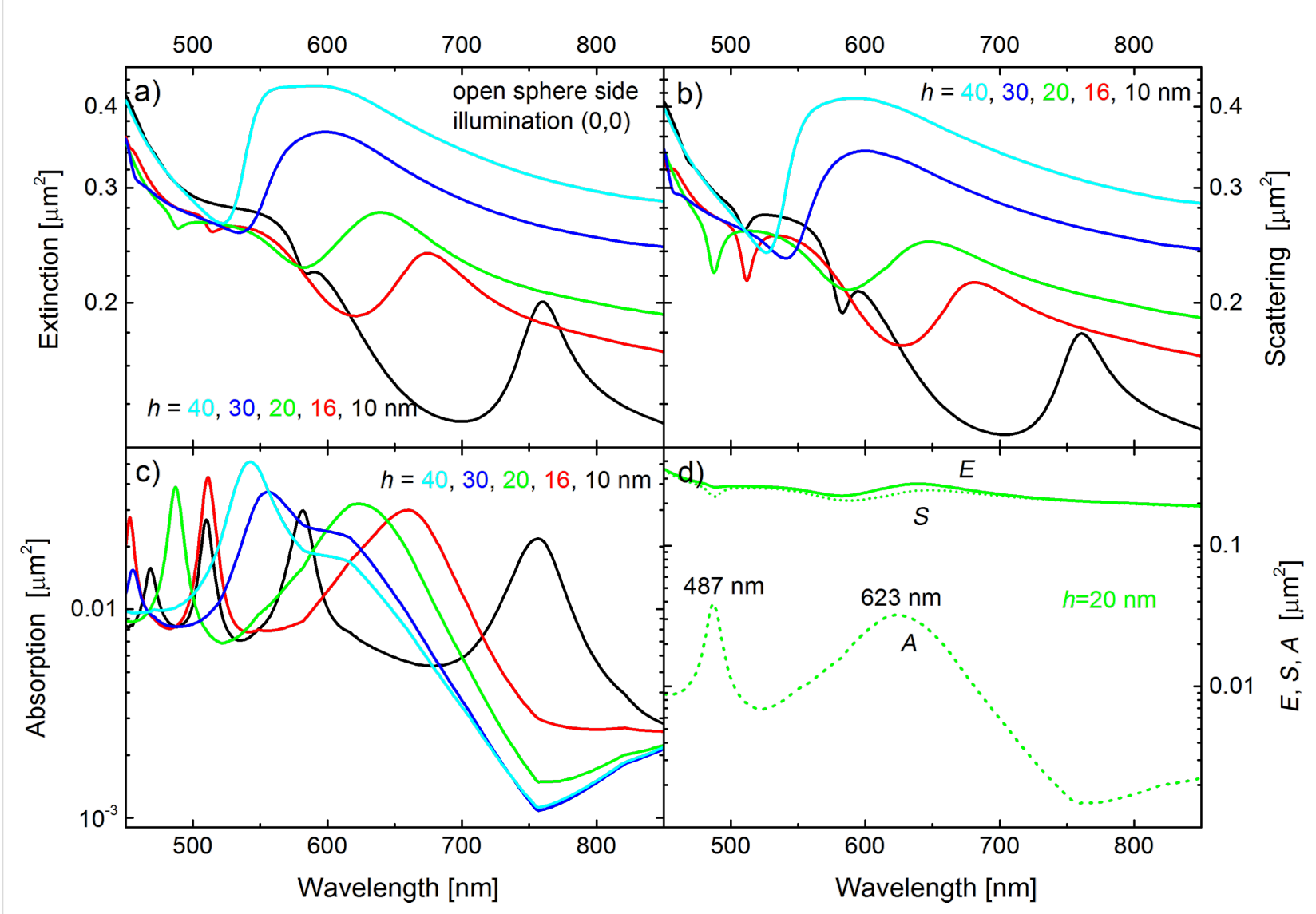

Figure 2: Cross sections of the (a) extinction, (b) scattering and (c) absorption of a PS sphere of diameter $d=390 \mathrm{~nm}$, without fluorophores, and capped with a Ag semi-shell of variable thickness $h$, as given by the color coding. The incident plane wave was oriented in $(0,0)$ direction, i.e., impinging from the open sphere side. The plasmonic resonances are most prominent in the absorption spectra. (d) compares the extinction ( $E$ ), scattering $(S)$ and absorption $(A)$ spectra for the specific shell thickness $h=20 \mathrm{~nm}$. 
tion peaks shifts to the red. For instance, the peak near $510 \mathrm{~nm}$ corresponds to the third resonance (counted from the long wavelength side) in the case of the $10 \mathrm{~nm}$ thick semi-shell (black curve), but to the second resonance in the case of $16 \mathrm{~nm}$ thickness (red curve). At a closer look, one can observe some minor peaks, sometimes masked as a shoulder of a main peak. The spectrum of resonances is quite rich and it is not straightforward to assign a specific $(l, m)$ mode to the peaks, which will become clearer later, when we use gain to sharpen the resonances. Only the longest-wavelength mode can safely be assigned to an $l=1$ mode.

Figure 3 shows the mode profiles in case of the semi-shell with $h=20 \mathrm{~nm}$. Specifically, it shows an $l=1$ and an $l=2$ mode with resonances in the absorption spectra at 623 and $487 \mathrm{~nm}$, respectively (c.f. Figure 2d). The upper panels in Figure 3 show the field enhancement $|\boldsymbol{E}| / E_{0}$, in which $E_{0}$ is the amplitude of the electric field in the incident plane wave. The images are cut in the $\mathrm{x}-\mathrm{z}$ plane, i.e., the plane spanned by the $\boldsymbol{k}$-vector (z-axis) and the polarization of the electric field (x-axis). A three dimensional representation of the near field enhancement just outside the semi-shell structure is given on the lower left of both panels. At $623 \mathrm{~nm}$, the plasmonic mode of the semi-shell can be derived from a pure dipolar resonance if the shell was not a semi-shell but a fully closed shell. Hence it is termed an $l=1$ resonance [11]. Because of the opening of the semi-shell (with respect to the closed shell), the $l=1$ resonances loose degeneracy and the mode depicted in Figure $3 \mathrm{a}$ is the transverse $l=1$ mode (the axial mode is not excitable by a plane wave in the $(0,0)$ direction) [16]. The mode at $487 \mathrm{~nm}$ is essentially an $l=2$ mode. Both modes are constructively coupled to the rim mode [11] and originate from the long-wavelength $l$ modes of the full shell [57]. The lower right graphs are polar graphs of the differential scattering cross section.

Figure 4 shows the extinction, scattering, and absorption cross sections of the $h=20 \mathrm{~nm}$ semi-shell structure for different illumination directions. The cross sections for the $(0,0)$ direction
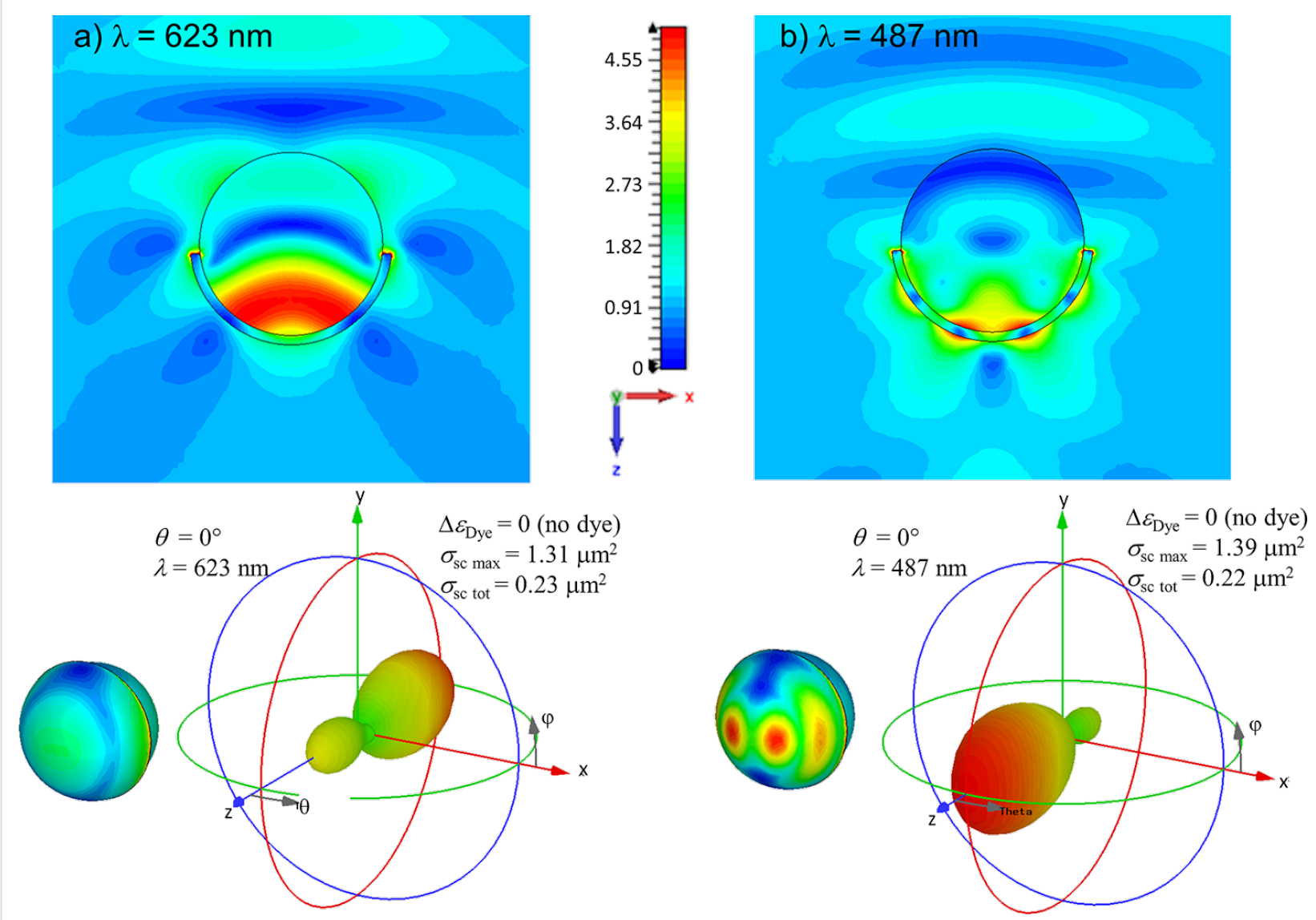

Figure 3: Mode characteristics of the two absorption maxima in Figure $2 \mathrm{~d}((0,0)$, i.e., sphere side illumination, $h=20 \mathrm{~nm})$, at $\lambda=623(\mathrm{a})$ and $487 \mathrm{~nm}$ (b). The upper images show the field enhancement $|E| / E_{0}$ in the propagation-polarization $x-z$ plane. The $3 D$ graphs on the lower left of (a) and (b) show $|E| / E_{0}$ in air near the surface of the semi-shell, i.e., the near-field just outside the structure. The mode at $623 \mathrm{~nm}$ is a "void like" dipolar mode $(I=1)$, while the mode at $487 \mathrm{~nm}$ is a $I=2$ mode. The $3 D$ spherical plots show the differential scattering cross sections on a linear scale. The maximum of the differential and the total $\sigma_{\mathrm{sc}}$ are given in the legends. 


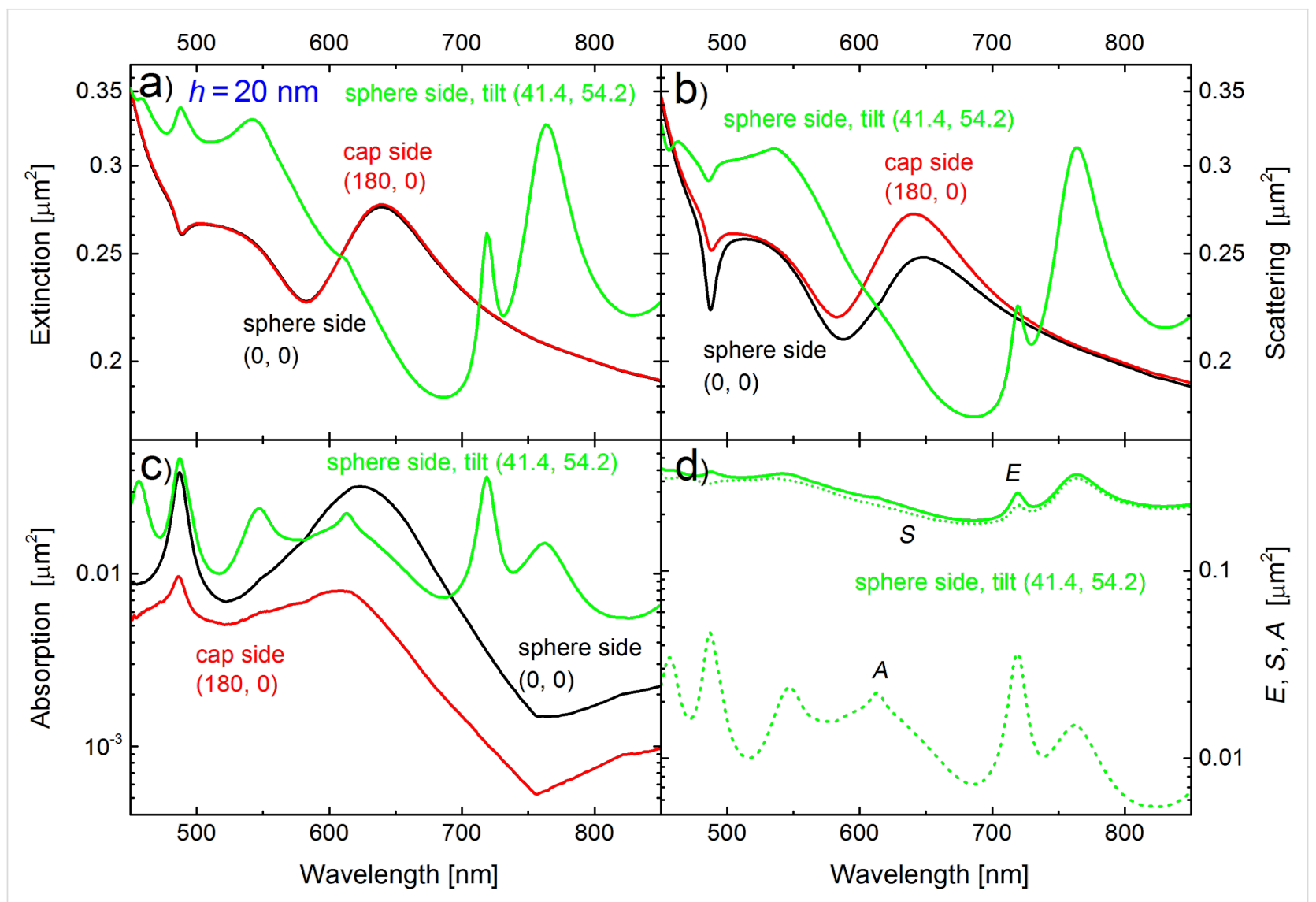

Figure 4: Dependence of the cross sections of the semi-shell structure with a core diameter $d=390 \mathrm{~nm}$ and a Ag thickness $h=20 \mathrm{~nm}$ on the direction of irradiation. Illumination from the open sphere side $(0,0)$ (black curve), or from the Ag-capped side $(180,0)$ (red curve), or from the sphere side with the plane wave impinging under the oblique angels $\theta=41.4^{\circ}, \varphi=54.2^{\circ}$ with the electric field in the $x-z$ plane (green curve). Other parameters and notations are the same as in Figure 1. Illumination under oblique angles leads to many more resonances because of the loss of degeneracy by cylindrical symmetry.

(illumination from the open sphere side) are redrawn from Figure $2 \mathrm{~d}$ for comparison (black curves). Red curves correspond to illumination from the silver capped side, along the negative z-axis $(180,0)$. Further, we compare both results with the cross sections obtained by an illumination under the oblique angles $(41.4,54.2)$. These angles sound a bit arbitrary, but they are equivalent to two consecutive rotations of the structure by $-30^{\circ}$ about the $\mathrm{x}-$ and $\mathrm{y}$ - axes, while leaving the incident wave intact. We see that the extinction cross sections of the sphere side $(0,0)$ and the cap side $(180,0)$ coincide, while the absorption and scattering cross sections differ in magnitude but not in the spectral shape. In contrast, there is a significant difference in the spectral shape for the $(41.4,54.2)$ orientation. Two new resonances appear (between 700 and $800 \mathrm{~nm}$ ), the main resonance at $640 \mathrm{~nm}$ disappears in the scattering spectrum (while still being visible at $623 \mathrm{~nm}$ in the absorption). A clear absorption peak appears at $545 \mathrm{~nm}$ where there was only a weak shoulder under $(0,0)$ or $(180,0)$ illumination, however, the $l=2$ absorption peak at $487 \mathrm{~nm}$ is similar to what we observed for the $(0,0)$ direction. This is an important finding, because we will spectrally overlap this $l=2$ absorption peak with the emission line of the dye molecules in the subsequent sections of the paper. Figure $4 \mathrm{~d}$ compares the extinction, scattering, and absorption cross sections for the case of the oblique incidence.

\section{Plasmons supported by gain}

In order to fine-tune the $l=2$ absorption peak to the emission line of the dye molecules, we plot the corresponding negative $\operatorname{Im}\left(\varepsilon_{\text {Sphere }}\right)$ into Figure $5 \mathrm{c}$ together with the absorption peaks of the semi-shells with thicknesses between 14 and $20 \mathrm{~nm}$. As the shell thickness varies, the $l=2$ absorption peak scans across the dye emission line. Figure $5 \mathrm{a}$ and Figure $5 \mathrm{~b}$ show the corresponding extinction and the scattering cross sections and Figure $5 \mathrm{~d}$ shows all three cross sections for the case of $h=16 \mathrm{~nm}$. For all calculations shown in Figure 5 we used a dielectric constant $\varepsilon_{\text {sphere }}$ defined in Equation 1, which includes the contribution of the dye molecules to the dielectric constant. The dotted line in Figure $5 \mathrm{c}$ represents $\operatorname{Im}\left(\varepsilon_{\text {Sphere }}\right)$. We used $\Delta \varepsilon_{\text {Dye }}=0.004$ and $\omega_{\mathrm{em}}$ matching the center of the emission line at $\lambda=513 \mathrm{~nm}$. We further used $\mathrm{A}=0$ and $\mathrm{E}=1$ in Equation 1, 


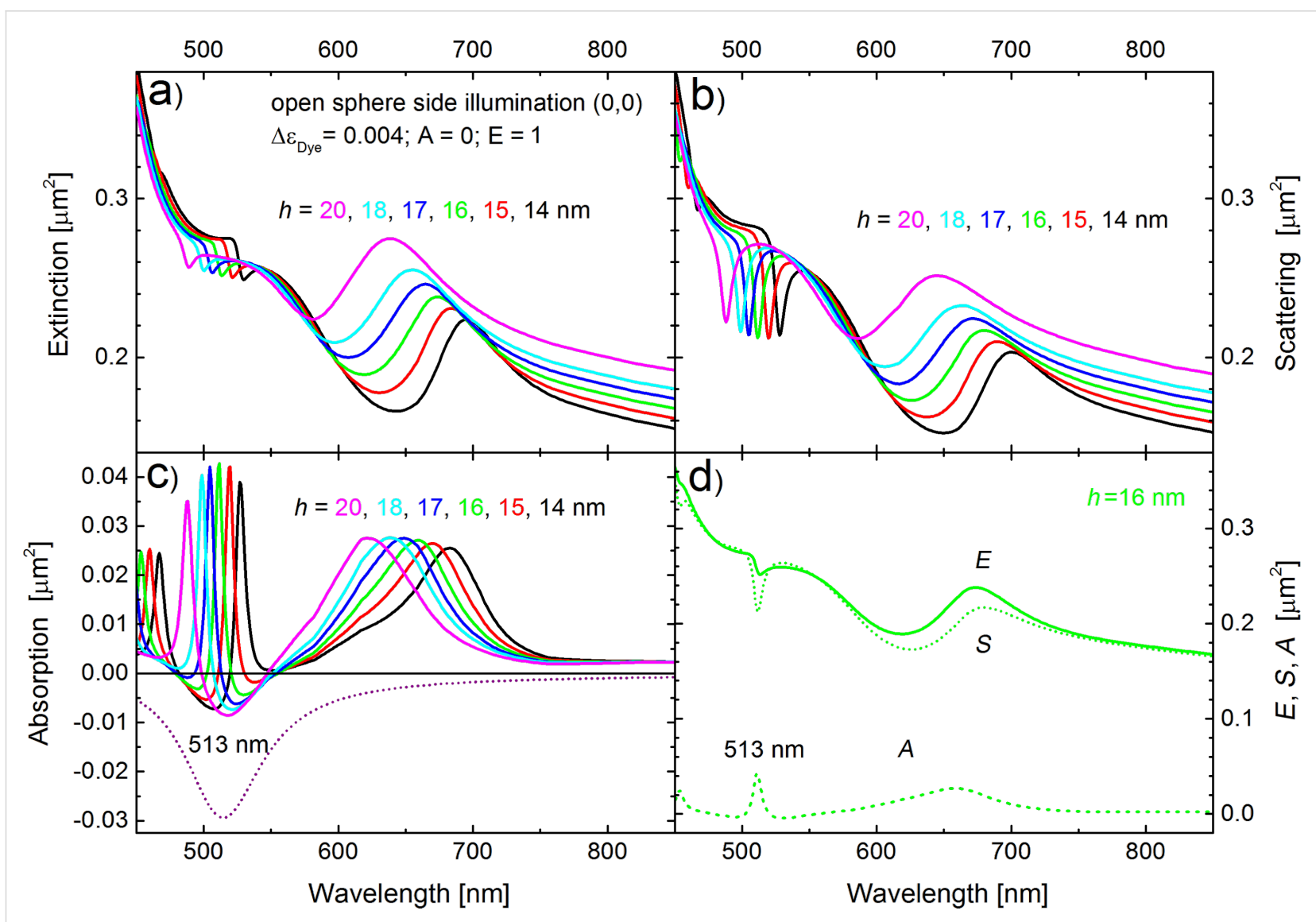

Figure 5: Fine-tuning of the Ag semi-shell resonances to the emission band of the dye. Parameters and the organization of panels a- $d$ are the same as in Figure 2 apart from the thickness $h$, color coded in the plots, and $\varepsilon_{\text {Sphere }}$ from Equation 1 , which now includes dye molecules. Dimensionless values of $\operatorname{Im}(\varepsilon$ Sphere $)$ are shown in panel (c) by a dotted curve.

which correspond to a full inversion of all dye molecules inside the PS spheres. This results in a negative absorption of our composite structure over a considerable spectral range. When $h=16 \mathrm{~nm}$ (green curves in Figure 5a-c) the $l=2$ absorption resonance perfectly coincides with the center of the emission line at $513 \mathrm{~nm}$. Besides, the absorption peak is the largest and the scattering is the lowest for this specific shell thickness as compared to the other thicknesses. We note in passing that the absorption cross sections in Figure $5 \mathrm{c}$ become negative for some wavelengths around the $l=2$ peak at $513 \mathrm{~nm}$ and for $\Delta \varepsilon_{\text {Dye }}=0.004$. We will discuss this in more detail at the end of this paper. Figure $5 \mathrm{~d}$ shows the extinction ( $E$, solid curve), scattering $(S$, dotted) and absorption $(A$, dashed) cross sections for the semi-shell thickness $h=16 \mathrm{~nm}$.

We now vary the amount of the active dye material inside the PS spheres in the simulations. Figure $6 \mathrm{a}-\mathrm{c}$ shows the extinction, scattering, and absorption cross sections, respectively, for the case of a semi-shell thickness $h=16 \mathrm{~nm}$, for which the emission line of the dye molecules and the absorption resonance of the semis-shell structures coincide. The strengths $\Delta \varepsilon_{\text {Dye }}$ of the absorption (a Lorentzian curve peaked at $468 \mathrm{~nm}$ ) and the gain (a Lorentzian curve peaked at $513 \mathrm{~nm}$ ) are color-coded as shown in the inset of Figure 6b. Hence, the cross sections of the undoped semi-shell structures are given by the black curve $\left(\Delta \varepsilon_{\text {Dye }}=0\right)$, the case of a purely absorbing commercially available PS sphere $\left(\Delta \varepsilon_{\text {Dye }}=0.004, \mathrm{~A}=1, \mathrm{E}=0\right.$ in Equation 1$)$ is given by the red line, the fully inverted case is given by the dark blue curve and the $50 \%$ inverted case by the green curve. The cyan, magenta, purple, and olive curves correspond to PS spheres that are hypothetically doped with fully inverted dye molecules to yield $\mathrm{E} \Delta \varepsilon_{\text {Dye }}=0.010,0.015,0.020$, and 0.030 , respectively. $\operatorname{Im}\left(\varepsilon_{\text {Sphere }}\right)$ is plotted into Figure $6 \mathrm{c}$ as a dotted curve for $\Delta \varepsilon_{\text {Dye }}=0.004, \mathrm{~A}=0$ and $\mathrm{E}=1$.

The onset of lasing is given by singularities in the absorption and scattering cross sections and occurs near $\Delta \varepsilon_{\text {Dye }}=0.0146$, which means at concentrations less than 3.7 times larger than available in commercial PS spheres. Hence, we conclude that lasing should be possible in custom made PS spheres with a moderately enhanced dye concentration. No lasing is observed yet at the commercially available gain strength of 


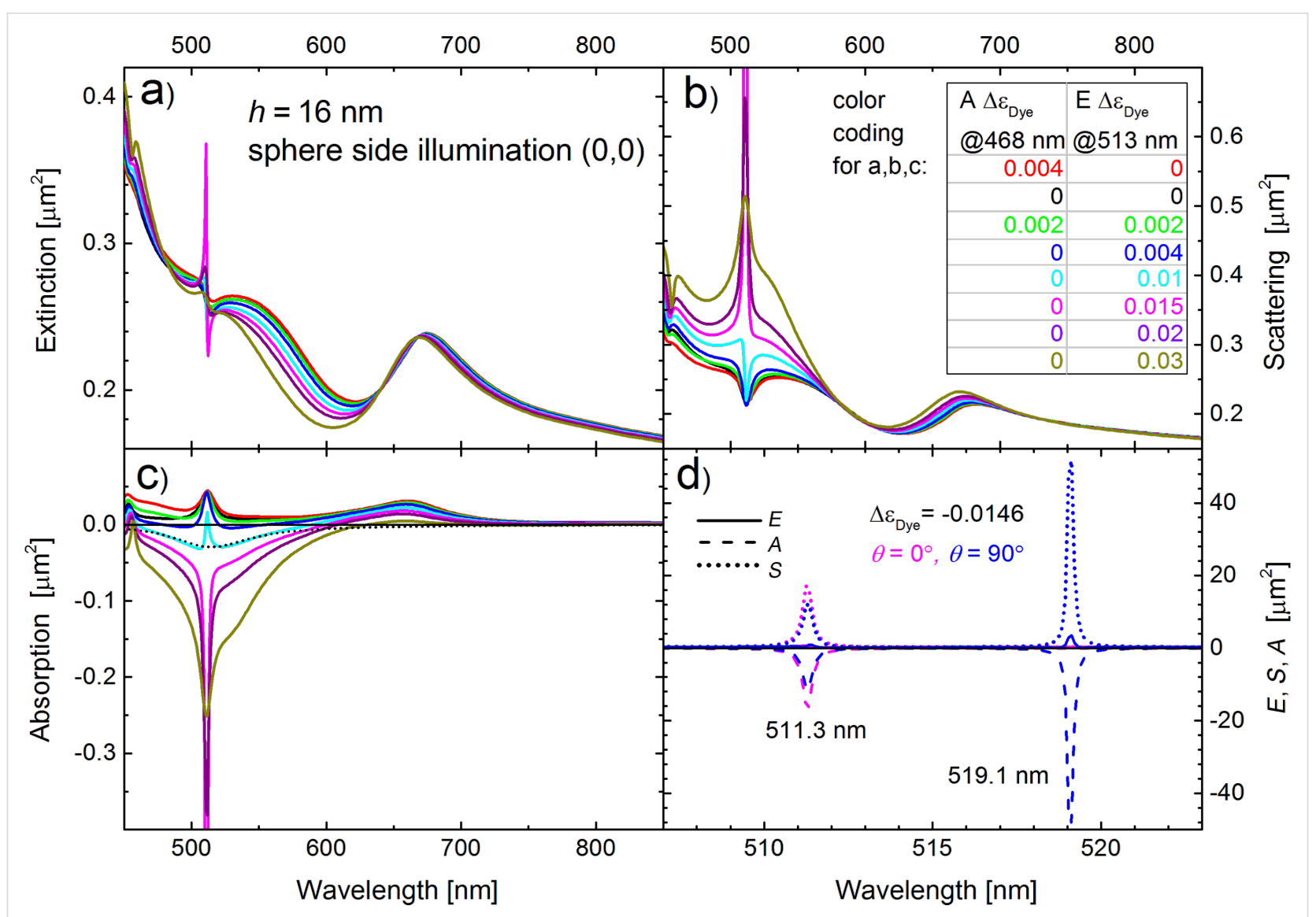

Figure 6: Approach to lasing for a semi-shell thickness of $h=16 \mathrm{~nm}$. Parameters and order of panels a,b,c are the same as in Figure 2. The strengths of the absorption and the gain (cf. Equation 1) are color-coded as shown in the inset of (b). The dimensionless $\operatorname{Im}\left(\varepsilon_{\text {Sphere }}\right)$ is shown in (c) by the dotted curve. Panel (d) shows a zoom of the extinction (solid curves), scattering (dotted) and absorption (dashed) spectra near the lasing threshold $\left(\Delta \varepsilon_{\text {Dye }}=0.0146, A=0, E=1\right)$ close to the $I=2$ resonance. The magenta lines refer to the sphere side irradiation $(0,0)$, while the blue lines are calculated for an illumination from the $x$-direction $\left(\theta=90^{\circ}, \varphi=0^{\circ}\right)$ so that the incident light is polarized in axial $z$-direction. In this case a second resonance appears near $519 \mathrm{~nm}$, in addition to the resonance near $511 \mathrm{~nm}$, which exists for both orientations.

$\Delta \varepsilon_{\text {Dye }}=0.004$, but the absorption cross section becomes slightly negative in the vicinity of the absorption peak (discussed in more detail below). When approaching laser threshold, the full width at half maximum (FWHM) of the scattering and absorption peaks goes to zero [51], which is an artifact of the purely classical calculation. Nevertheless, this singularity in cross sections indicates the transition from an amplifier to self-sustained lasing. A CW operation of a nano-spaser above the threshold $\left(\Delta \varepsilon_{\text {Dye }}>0.0146\right)$ is possible only with continuous pumping, in which the field strength and linewidth are dictated by the gain saturation and spontaneous emission noise $[36,56,58]$. We do not discuss the thermal stability of such a system, which is strongly material-dependent.

In panels a-c of Figure 6 , only the $(0,0)$ illumination direction (open sphere side) is considered. Figure $6 \mathrm{~d}$ compares the $(0,0)$ direction (magenta curves) with the $(90,0)$ direction (blue curves), in which the electric field is now polarized along the $\mathrm{z}$-axis and hence allows for the excitation of axial cap plas- mons. Note that the abscissa is zoomed in to span 507 to $523 \mathrm{~nm}$, and the ordinate is enlarged to span -50 to $55 \mu \mathrm{m}^{2}$ in order to show the almost diverging peaks for $\Delta \varepsilon_{\text {Dye }}=0.0146$. The solid lines in Figure $6 \mathrm{~d}$ are the extinction cross sections, the dashed lines the absorption and the dotted lines the scattering cross sections. One clearly sees that the resonance at $511.3 \mathrm{~nm}$ is strongly excited in both cases, for $(0,0)$ and for $(90,0)$ illumination. However, a second resonance at $519.1 \mathrm{~nm}$ appears for the $(90,0)$ illumination, which is not excitable with $(0,0)$ illumination for symmetry reasons. We note that only because of the substantially narrowed spectral lines (due to gain), both resonances are discernible. Without gain, both peaks merge because of the relatively broad width of the damped plasmonic resonances (cf. Figure $5 \mathrm{~b}$ and Figure $5 \mathrm{c}$ ). The marginal spectral shift from $513 \mathrm{~nm}$ for $\Delta \varepsilon_{\text {Dye }}=0.004$ (Figure $5 \mathrm{~d}$ ) to $511.3 \mathrm{~nm}$ in case of the lasing gain of $\Delta \varepsilon_{\text {Dye }}=0.015$ can be attributed to slight differences in the dielectric constant (Equation 1) of the PS sphere near the resonance for different gain levels, as well as to numerical discretization issues. 
In order to investigate the spasing modes in more detail, we plot the differential scattering cross sections in Figure 7 for the case of $(0,0)$ illumination and for the cases of no dye molecules (Figure 7a), a currently commercially available concentration (Figure 7b) and for a dye doping close to the lasing threshold (Figure 7c). We plot the cross sections close to the resonances at $513 \mathrm{~nm}$ (Figure 7a and Figure 7b) and $511.3 \mathrm{~nm}$ (Figure 7c). While the differential scattering cross sections in case of undoped spheres (Figure 7a) and spheres doped with available concentrations (Figure 7b) look very similar and show Mie-type forward scattering, the pattern completely changes near the lasing threshold (Figure 7c). Here, the light is scattered predominantly in two lobes in the $\mathrm{x}-\mathrm{z}$ plane, which point backward in $z$-direction (remember that the incident wave is along the positive $\mathrm{z}$-direction). Further, the total scattering cross section is approx. $0.2 \mu \mathrm{m}^{2}$ in case of no dye doping and commercially available doping, but increases significantly to approx. $17.1 \mu \mathrm{m}^{2}$ close to the lasing threshold [29].

Figure 8 shows the (near-)field enhancement $|\boldsymbol{E}| / E_{0}$ close to the semi-shell structure and the differential scattering cross sections in the case of $(90,0)$ illumination, for a near-threshold gain $(\mathrm{A}=0, \mathrm{E}=1)$ and for the two resonances (cf. Figure 6d, blue curves). Figure $8 \mathrm{a}$ and Figure $8 \mathrm{~b}$ are calculated at $511.3 \mathrm{~nm}$, close to the first spasing peak, and Figure $8 \mathrm{c}$, and Figure $8 \mathrm{~d}$ are calculated at $519.1 \mathrm{~nm}$, close to the second peak that cannot be excited with $(0,0)$ illumination. While the near-field distribution for the peak at $511.3 \mathrm{~nm}$ (Figure 8a) looks very similar (but with much stronger fields) to the case of the undoped semi-shell structure (Figure $3 \mathrm{~b}$ ), the near field distribution at $519.1 \mathrm{~nm}$ (Figure 8c) looks very different and resembles an $(l=3, m=3$ ) orbital. Clearly, this eigen-resonance is easily overlooked in experiments, in which semi-shells with insufficient or no gain are investigated because it would be hidden within the natural width of the $l=2$ resonance at $513 \mathrm{~nm}$, and it can also be overlooked in numerical calculations because the wavelength stepping needs to be small enough not to miss it.

The mode at $511.3 \mathrm{~nm}$ can be excited with both the $(0,0)$ and the $(90,0)$ illumination directions. Hence we can compare the scattering diagrams for both cases at a gain close to threshold (Figure $7 \mathrm{c}$ and Figure $8 \mathrm{~b}$, respectively). The total scattering cross section is larger in the case of the $(0,0)$ illumination than for the $(90,0)$ direction $\left(17.1 \mu \mathrm{m}^{2}\right.$ versus $12.3 \mu \mathrm{m}^{2}$, respectively), but the angular distributions look very similar. In stark contrast, the $519.1 \mathrm{~nm}$ mode, which is excitable only through $(90,0)$ illumination, shows a decisively different far-field scattering distribution (Figure 8d). It is also worth mentioning that the near-field distributions (Figure 3b, Figure 8a, Figure 8c) look very different to the far-field differential cross sections (Figure 7c, Figure 8b, Figure 8d).
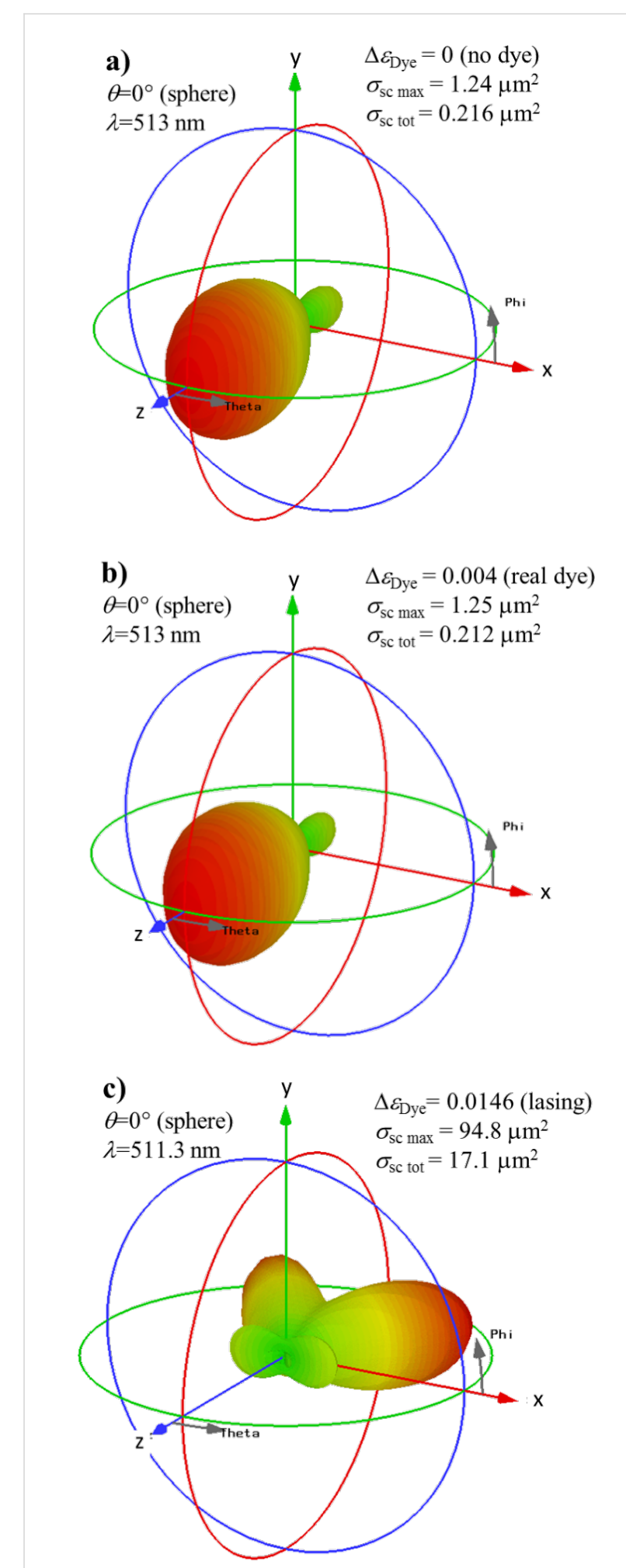

Figure 7: Differential scattering cross section $\sigma_{\mathrm{sc}}$, linear scale, for resonant semi-shells with thickness $h=16 \mathrm{~nm}$ at $\lambda \approx 513 \mathrm{~nm}$. The irradiation is from the $(0,0)$ direction; the strength of the emission (gain, $A=0, E=1$ ) is (a) $\Delta \varepsilon_{\text {Dye }}=0$ (no dye molecules at all), (b) 0.004 (commercially available dye concentration and total inversion) and (c) 0.0146 (lasing condition). The maximum of the differential and the total $\sigma_{\mathrm{sc}}$ are given in the legends. 


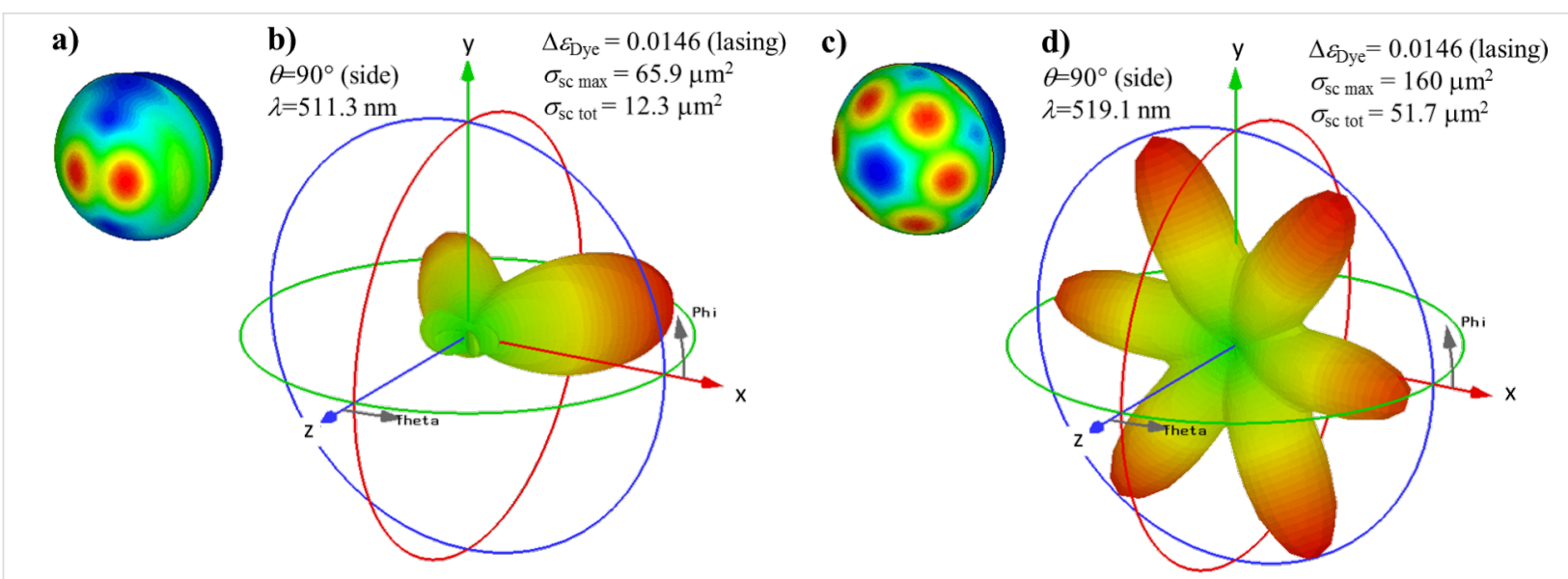

Figure 8: (a,c) Field enhancement $|E| / E_{0}$ for two modes with gain close to the lasing threshold. The z-polarized incoming plane wave propagates in x-direction $\left(\theta=90^{\circ}, \varphi=0^{\circ}\right)$. The two wavelengths $511.3 \mathrm{~nm}$ (a) and $519.1 \mathrm{~nm}$ (c) are close to the two peaks in Figure $6 \mathrm{~d}$. The semi-shell thickness $h=16 \mathrm{~nm}$, and a gain strength $\Delta \varepsilon_{\text {Dye }}=0.0146$ (near lasing condition) are assumed. (b,d) Differential scattering cross sections $\sigma_{\mathrm{sc}}$, linear scale. The maxima of differential and total $\sigma_{\mathrm{sc}}$ are given in the legends.

This observation becomes even more prominent when we look at a semi-shell of a slightly increased thickness of $h=20 \mathrm{~nm}$ instead of $16 \mathrm{~nm}$. Figure 9a shows the extinction (solid lines), scattering (dotted lines) and absorption cross sections (dashed lines) for illumination from the $(0,0)$ direction (magenta lines) and the $(90,0)$ direction (blue lines) and hence Figure 9a directly corresponds to Figure 6d. Again, a shorter wavelength resonance, this time near $491 \mathrm{~nm}$, is excitable for both illumination directions $(0,0)$ and $(90,0)$, while the longer wavelength "axial" resonance, this time at $497.5 \mathrm{~nm}$, is only excitable in case of $(90,0)$ illumination. Note, that appreciably larger gain of approximately $\Delta \varepsilon_{\text {Dye }}=0.0217$ is needed in case of the $h=20 \mathrm{~nm} \mathrm{Ag} \mathrm{semi-shell} \mathrm{to} \mathrm{reach} \mathrm{the} \mathrm{lasing} \mathrm{threshold} \mathrm{as}$ compared to only $\Delta \varepsilon_{\text {Dye }}=0.0146$ in case of the thinner shell of $h=16 \mathrm{~nm}$. This is explained by two facts: a) larger losses in a thicker metal, and b) the absorption peak of the $h=16 \mathrm{~nm}$ shell coincides with the Lorentzian gain profile (cf. Figure $5 \mathrm{c}$ ), while the peak of the $h=20 \mathrm{~nm}$ shell does not. Panels (b,c), (d,e), and $(\mathrm{g}, \mathrm{f})$ of Figure 9 show the near-field distributions and the farfield scattering for the semi-shell structure with $h=20 \mathrm{~nm}$ for the following conditions: $(0,0)$ illumination at $490.7 \mathrm{~nm}(\mathrm{~b}, \mathrm{c})$, $(90,0)$ illumination at $490.7 \mathrm{~nm}(\mathrm{~d}, \mathrm{e})$ and $(90,0)$ illumination at $497.5 \mathrm{~nm}(\mathrm{f}, \mathrm{g})$. Note that in case of (b-e) $\Delta \varepsilon_{\text {Dye }}=0.0217$ was used instead of 0.020 as in (a) and $(f, g)$ because the lasing thresholds for the $497.5 \mathrm{~nm}$ resonance and the $490.7 \mathrm{~nm}$ resonance turned out to be slightly different. These graphs should be compared to the respective graphs for the $h=16 \mathrm{~nm} \mathrm{Ag} \mathrm{semi-}$ shells, namely Figure $7 \mathrm{c}$ for the $(0,0)$ illumination and Figure 8 for the $(90,0)$ illumination. We start with the discussion of the long-wavelength resonances $519.1 \mathrm{~nm}$ (Figure 8c,d) and $497.5 \mathrm{~nm}$ (Figure 9f,g), which can only be excited with the axially polarized $(90,0)$ illumination. The near-field distributions (Figure $8 \mathrm{c}$ and Figure 9f) as well as the differential scat- tering cross sections (Figure 8d and Figure 9g) look very similar. Only the relative strengths of the six lobes show some differences. The same tendency is observed for the "transverse" shorter-wavelength resonances at 511.3 and $491 \mathrm{~nm}$ (compare Figures $7 \mathrm{c}, 8 \mathrm{a}, \mathrm{b}$ and $9 \mathrm{~b}-\mathrm{e}$ ). We would like to note that the patterns of the differential cross sections change dramatically within a narrow range of parameters, which can be easily overlooked in the experiments as well as in the simulations. The present scattering diagrams differ strongly from the more symmetric ones reported in [51], in which smaller and thinner semi-shell structures were simulated. (The results from this report are fully reproducible by our numerical framework). All this corroborates the observation that one cannot easily foretell the pattern of the differential scattering cross sections from the appearance of the plasmonic eigenmode.

Lastly, let us discuss, which spectroscopic footprints can be expected from a commercially available dye-doping level that yields $\Delta \varepsilon_{\text {Dye }}=0.004$. Obviously, one cannot expect spasing, because a 3.7 times larger dye concentration is mandatory for that in the current geometry. However, already at moderate doping levels, some unusual spectroscopic signals should be observable. Figure 10 compares the differential cross sections for semi-shell thicknesses $h=20 \mathrm{~nm}$ (solid curves) and $h=16 \mathrm{~nm}$ (dashed curves), at dye concentrations that can be found in commercially available PS spheres. All other parameters are the same as in Figure 2. The most striking result is that for $h=20 \mathrm{~nm}$ the absorption becomes negative near $513 \mathrm{~nm}$, and for $h=16 \mathrm{~nm}$ it becomes negative near 500 and $530 \mathrm{~nm}$. This means that the structures show an overcompensation of absorption at these wavelengths but no lasing yet. Changes in absorption for such concentrations are actually more pronounced for the off-resonance thickness $h=20 \mathrm{~nm}$. Further, the 


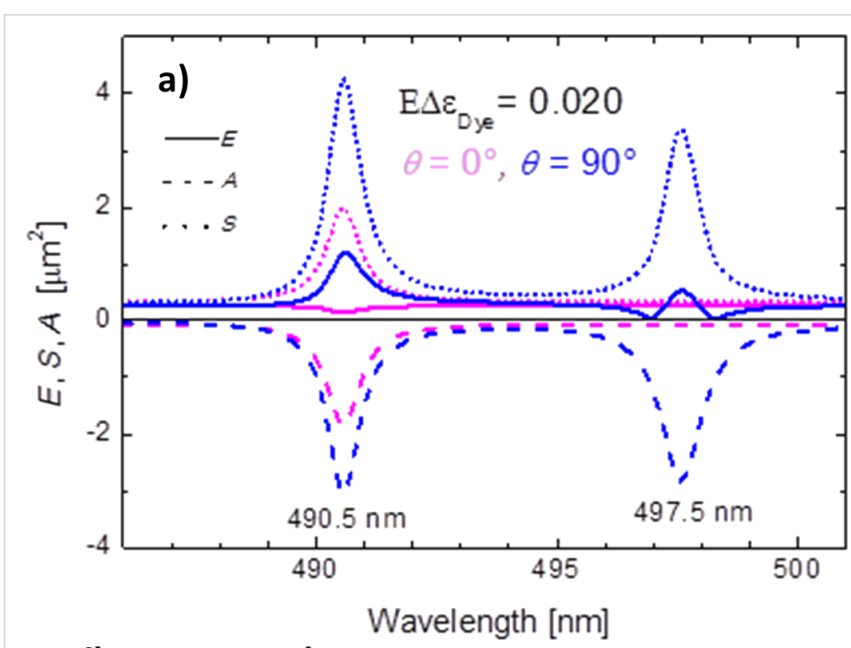

d)

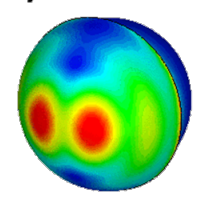

e)

$\theta=90^{\circ}$ (side) $\quad$ y $\quad \Delta \varepsilon_{\text {Dye }}=0.0217$ (lasing)

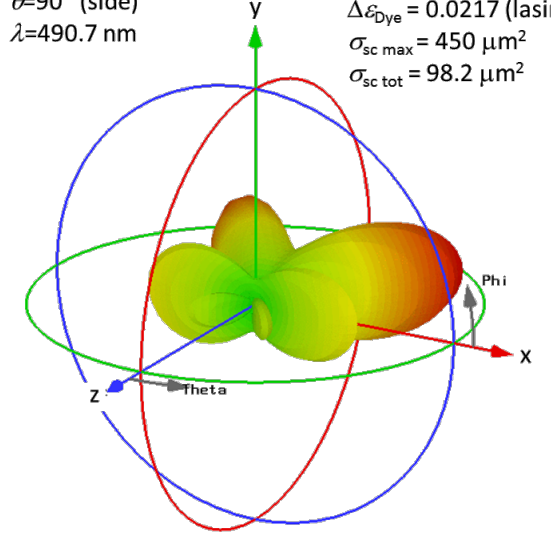

b)

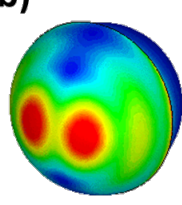

c)

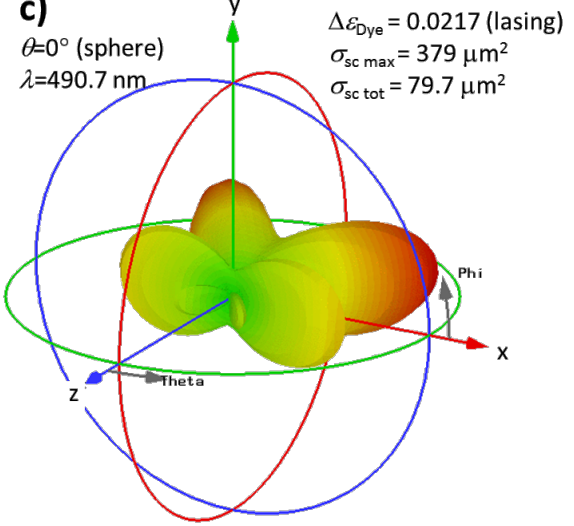

f)

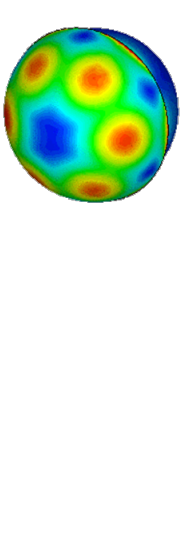

g)

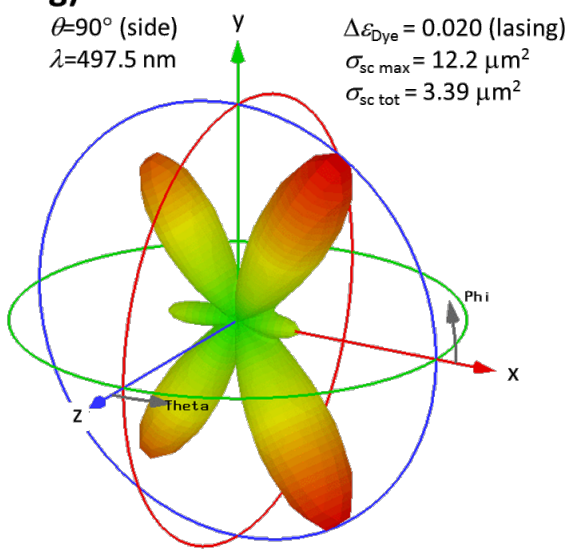

Figure 9: (a) Cross sections in case of a semi-shell of thickness $h=20 \mathrm{~nm}$ near the lasing threshold of $\Delta \varepsilon_{\text {Dye }}=0.020, \mathrm{~A}=0$, $\mathrm{E}=1$, extinction (solid lines), scattering (dotted lines) and absorption cross sections (dashed lines) for illumination from the $(0,0)$ direction (magenta lines) or $(90,0)$ direction (blue lines). (b-g) Near-field distribution and differential scattering cross sections; (b,c): for the $491 \mathrm{~nm}$ resonance and the $(0,0)$ illumination; (d,e): for the $491 \mathrm{~nm}$ resonance and the $(90,0)$ illumination; and $(\mathrm{f}, \mathrm{g})$ : for the $497.5 \mathrm{~nm}$ resonances and $(90,0)$ direction. Note that in the case of $(\mathrm{b}-\mathrm{e})$ $\Delta \varepsilon_{\text {Dye }}=0.0217$ was used instead of 0.020 as in $(a)$ and $(f, g)$, see main text.

features in the scattering spectrum are sharpened by gain and so do the (positive) peaks in the absorption spectrum at wavelength ranges, at which the absorption is not yet overcompensated, for instance the peaks at $487 \mathrm{~nm}$ in the case of the $h=20 \mathrm{~nm}$ semi-shells (Figure $10 \mathrm{c}$, solid lines). Finally, Figure 10d compares all cross sections on one plot for the full inversion of the dye molecules with realistic concentration for both thicknesses $h=20 \mathrm{~nm}$ (dark blue), and $h=16 \mathrm{~nm}$ (cyan). In Figure 10d the solid curves refer to extinction $(E)$, dotted to scattering $(S)$ and dashed to absorption $(A)$.

\section{Conclusion}

Dye-doped polystyrene spheres capped with a thin semi-shell of silver show rich spectra of absorption, scattering and extinction, which contain many peaks and shoulders. Without gain, i.e., as long as the dye molecules are not pumped, the spectra are broad and hiding some of the eigenmodes. However, these become clearly observable when the gain is switched on, which leads to a compensation of absorption and hence sharpens the plasmon resonances. Specifically, we showed that a mode of $l=3, m=3$ symmetry is only $8 \mathrm{~nm}$ away from a $l=2$ mode. This tiny difference can certainly not be resolved without gain-narrowing of plasmonic resonances. We deliberately focused on the discussion of the gain that is required for spectral sharpening and compared the required gain to the gain available in commercially available polystyrene spheres. We find that for the investigated geometries spasing requires gain levels less than 3.7 times higher than those in commercially available dyedoped spheres. Furthermore, the directionality of the differential scattering cross section changes with the amount of gain, depends on the fine details of the structure geometry and is sensitive to the direction of illumination. All this demands a high manufacturing accuracy of semi-shell based spasers.

With gain, some "conventional intuition" might become wrong. For example that lower multipolar modes are usually the 


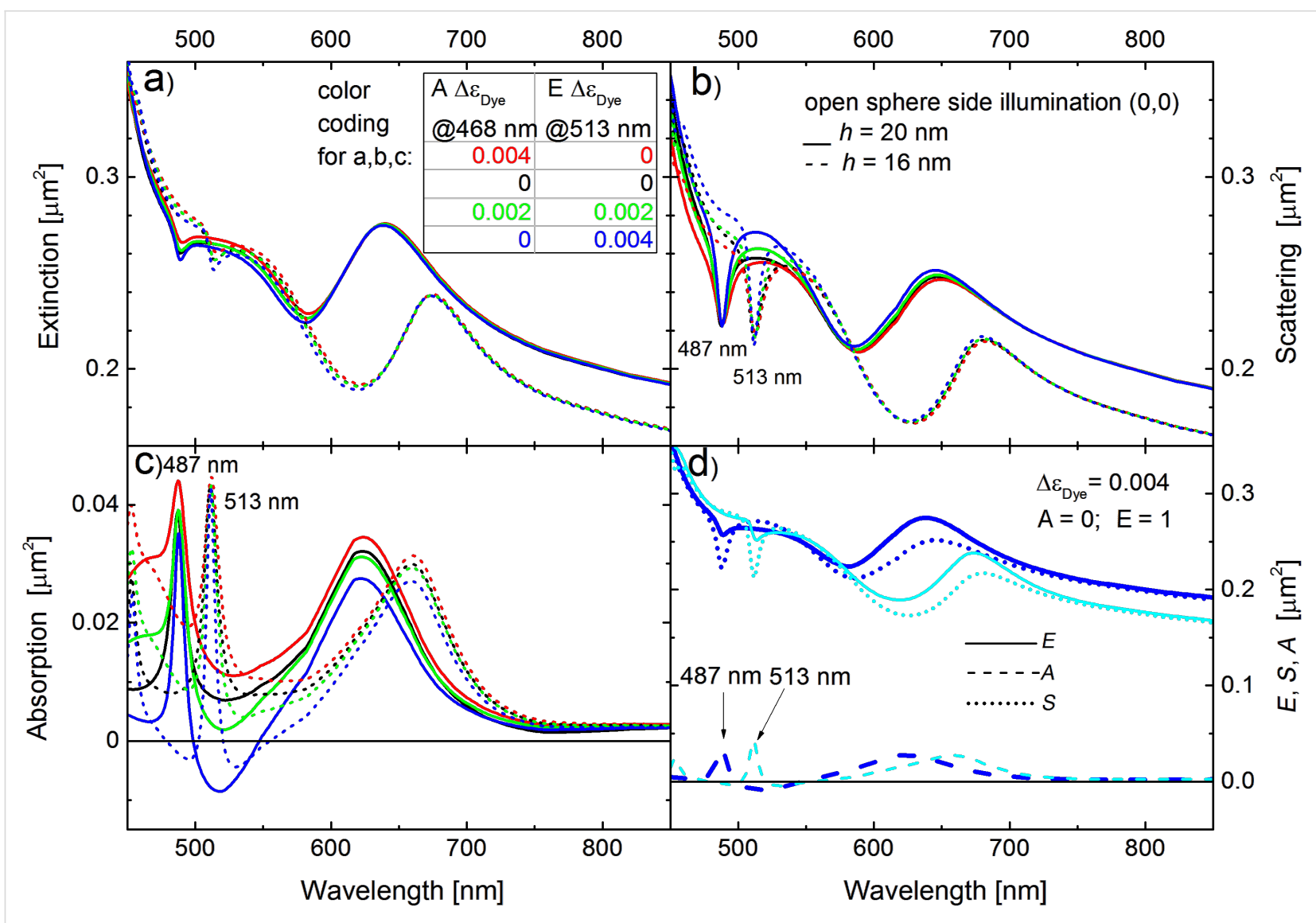

Figure 10: Differential cross sections for semi-shell thicknesses $h=20 \mathrm{~nm}$ (solid curves) and $h=16 \mathrm{~nm}$ (dashed curves), at dye concentrations as in commercially available PS spheres. All other parameters are the same as in Figure 2. For $h=20 \mathrm{~nm}$ the absorption becomes negative near $513 \mathrm{~nm}$, while for $h=16 \mathrm{~nm}$ it becomes negative near 500 and $530 \mathrm{~nm}$. The structures show overcompensation of absorption at these wavelengths but no lasing yet. (d) Comparison of all cross sections on one plot for $\Delta \varepsilon_{\text {Dye }}=0.004, \mathrm{~A}=0.004, \mathrm{E}=1$ for both thicknesses $h=20 \mathrm{~nm}$ (dark blue), and $h=16 \mathrm{~nm}$ (cyan).

stronger ones. This intuition is based on the expansion into powers of the Mie-parameter $(a / \lambda)$ where $a$ is the typical structure size. This expansion still holds. However, when the denominator in one of the scattering/absorption terms goes to zero, this term dominates, irrespectively of the power of $(a / \lambda)$ associated with it. Some of the higher modes may dominate simply because they best match the spectral bandwidth of the gain media.

\section{Numerical}

Here we summarize several subtleties, crucial for reliable simulations. Conventional finite difference time domain (FDTD) approaches require an analytical approximation of the dispersion of the metal dielectric constant [59], which is not always satisfactory. Active media with optical gain may introduce instabilities into FDTD codes, unless auxiliary differential equations with gain saturation are used [60]. We tackle these difficulties using the (mainly iterative) frequency domain solver of the CST MWS ${ }^{\circledR}$ software with open boundaries, realized by perfectly matched layers (PML, 10 mesh layers). The computa- tional domain (without PML) is a cube with a side of about $1 \mu \mathrm{m}$. For excitation, a linearly polarized plane wave is applied. No symmetries were used in order to make the numerical framework fully suitable for oblique incidence under angles $(\theta \neq 0, \varphi \neq 0)$ and for asymmetric structures.

Special care has to be taken of sharp edges and vertices leading to numerical hot spots, which are, however, unrealistic because in experiments all vertices will show some natural rounding and even if very large curvatures could be realized, ultra-high field enhancement is unphysical on a scale below $1 \mathrm{~nm}$ because of nonlocality and quantum effects $[61,62]$. We used blended edges with radii of curvature $R_{\text {blend }}=5 \mathrm{~nm}$.

The numerically prepared Lorentzian gain can be read into the CST software package as a list of $\varepsilon$ values only if $\varepsilon$ " is artificially made positive. Afterwards, the properties of the material can be manually reverted to $\varepsilon^{\prime \prime}<0$ in the "history list". The CST software calculates scattering and absorption cross sections $\sigma_{\mathrm{sc}}$, $\sigma_{\mathrm{ab}}$ by using internal algorithms. However, the absorption 
calculation fails for the active structures, when $\sigma_{\mathrm{ab}}$ becomes negative. For this reason, a Power Flow Monitor through a spherical face surrounding the structure was created, and the integral power flow through it was recalculated into $\sigma_{\mathrm{ab}}$. The extinction cross section is always defined as $\sigma_{\mathrm{ex}}=\sigma_{\mathrm{sc}}+\sigma_{\mathrm{ab}}$, irrespectively of the sign of $\sigma_{\mathrm{ab}}$. Far-field patterns are calculated by the internal CST routines, which project the fields on the sides of the bounding box into the far-fields via fast Fourier transform.

Adaptive meshing is a must for the accurate representation of the fields in the regions where their gradients are high [63], though it affects less such global characteristics as $\sigma_{\mathrm{ex}}, \sigma_{\mathrm{sc}}$ and $\sigma_{\mathrm{ab}}$. For time-efficient calculations distributed computing (DC) and parallelization capabilities of the CST package were used, usually with six solver servers, each calculating its own frequency point independently in parallel, and threaded over 2 CPUs. In a typical run, 12 employed CPUs needed a walltime of about $2 \mathrm{~h}$ for the full spectra with about 300 frequency points. Usually 10 adaptive mesh runs were sufficient, resulting in $80-90 \mathrm{kcells}$, with the cell edge length varying between $\approx 1 \mathrm{~nm}$ near (smoothened) sharp metallic features and $\approx 50 \mathrm{~nm}$ in the free air regions.

\section{Acknowledgements}

We are grateful to Dr. Ilari Hänninen (CST Darmstadt) and DI Johann Messner (Scientific Computing Center, Johannes Kepler University, Linz) for many helpful advises on numerical issues, specifically on software parallelization. Financial support from the European Research Council is acknowledged within the FP7 Program via the ERC Starting Grant (Active NP, 257 158).

\section{References}

1. Kreibig, U.; Vollmer, M. In Optical Properties of Metal Clusters; Toennies, J. P., Ed.; Springer Series in Materials Science, Vol. 25; Springer: Berlin, 1995.

2. Zhou, H. S.; Honma, I.; Komiyama, H.; Haus, J. W. Phys. Rev. B 1994, 50, 12052-12056. doi:10.1103/PhysRevB.50.12052

3. Averitt, R. D.; Sarkar, D.; Halas, N. J. Phys. Rev. Lett. 1997, 78, 4217-4220. doi:10.1103/PhysRevLett.78.4217

4. Takei, H. J. Vac. Sci. Technol., B 1999, 17, 1906-1911. doi:10.1116/1.591066

5. Love, J. C.; Gates, B. D.; Wolfe, D. B.; Paul, K. E.; Whitesides, G. M. Nano Lett. 2002, 2, 891-894. doi:10.1021/nl025633।

6. Liu, J.; Maaroof, A. I.; Wieczorek, L.; Cortie, M. B. Adv. Mater. 2005, 17, 1276-1281. doi:10.1002/adma.200500035

7. Coyle, S.; Netti, M. C.; Baumberg, J. J.; Ghanem, M. A.; Birkin, P. R.; Bartlett, P. N.; Whittaker, M. Phys. Rev. Lett. 2001, 87, 176801. doi:10.1103/PhysRevLett.87.176801

8. Charnay, C.; Lee, A.; Man, S.-Q.; Moran, C. E.; Radloff, C.; Bradley, R. K.; Halas, N. J. J. Phys. Chem. B 2003, 107, 7327-7333. doi:10.1021/jp034309r

9. Lassiter, J. B.; Knight, M. W.; Mirin, N. A.; Halas, N. J. Nano Lett. 2009, 9, 4326-4332. doi:10.1021/n19025665
10. Ye, J.; Van Dorpe, P.; Van Roy, W.; Lodewijks, K.; De Vlaminck, I.; Maes, G.; Borghs, G. J. Phys. Chem. C 2009, 113, 3110-3115. doi:10.1021/jp8072409

11. Cole, R. M.; Baumberg, J. J.; Garcia de Abajo, F. J.; Mahajan, S.; Abdelsalam, M.; Bartlett, P. N. Nano Lett. 2007, 7, 2094-2100. doi:10.1021/nI0710506

12. Cortie, M.; Ford, M. Nanotechnology 2007, 18, 235704. doi:10.1088/0957-4484/18/23/235704

13. Wang, Q.; Tang, C.; Chen, J.; Zhan, P.; Wang, Z. Opt. Express 2011, 19, 23889-23900. doi:10.1364/OE.19.023889

14. Lacharmoise, P. D.; Tognalli, N. G.; Goñi, A. R.; Alonso, M. I.; Fainstein, A.; Cole, R. M.; Baumberg, J. J.; Garcia de Abajo, J.; Bartlett, P. N. Phys. Rev. B 2008, 78, 125410. doi:10.1103/PhysRevB.78.125410

15. Zhang, Y.; Barhoumi, A.; Lassiter, J. B.; Halas, N. J. Nano Lett. 2011, 11, 1838-1844. doi:10.1021/nl2008357

16. King, N. S.; Li, Y.; Ayala-Orozco, C.; Brannan, T.; Nordlander, P.; Halas, N. J. ACS Nano 2011, 5, 7254-7262. doi:10.1021/nn202086u

17. Ding, B.; Hrelescu, C.; Arnold, N.; Isic, G.; Klar, T. A. Nano Lett. 2013, 13, 378-386. doi:10.1021/nl3035114

18. Himmelhaus, M.; Takei, H. Sens. Actuators, B 2000, 63, 24-30. doi:10.1016/S0925-4005(99)00393-7

19. Lal, N. N.; Soares, B. F.; Sinha, J. K.; Huang, F.; Mahajan, S.; Bartlett, P. N.; Greenham, N. C.; Baumberg, J. J. Opt. Express 2011, 19, 11256-11263. doi:10.1364/OE.19.011256

20. Dunbar, R. B.; Pfadler, T.; Lal, N. N.; Baumberg, J. J.; Schmidt-Mende, L. Nanotechnology 2012, 23, 385202. doi:10.1088/0957-4484/23/38/385202

21. Mahajan, S.; Richardson, J.; Brown, T.; Bartlett, P. N. J. Am. Chem. Soc. 2008, 130, 15589-15601. doi:10.1021/ja805517q

22. Mahajan, S.; Cole, R. M.; Soares, B. F.; Pelfrey, S. H.; Russell, A. E.; Baumberg, J. J.; Bartlett, P. N. J. Phys. Chem. C 2009, 113, 9284-9289. doi:10.1021/jp900661u

23. Steuwe, C.; Kaminski, C. F.; Baumberg, J. J.; Mahajan, S. Nano Lett. 2011, 11, 5339-5343. doi:10.1021/nl202875w

24. Sudarkin, A. N.; Demkovich, P. A. Sov. Phys. - Tech. Phys. 1989, 34, 764-766.

25. Klar, T. A.; Kildishev, A. V.; Drachev, V. P.; Shalaev, V. M. IEEE J. Sel. Top. Quantum Electron. 2006, 12, 1106-1115. doi:10.1109/JSTQE.2006.880597

26. Xiao, S.; Drachev, V. P.; Kildishev, A. V.; Ni, X.; Chettiar, U. K.; Yuan, H.-K.; Shalaev, V. M. Nature 2010, 466, 735-738. doi:10.1038/nature09278

27. Campione, S.; Capolino, F. Nanotechnology 2012, 23, 235703. doi:10.1088/0957-4484/23/23/235703

28. Campione, S.; Albani, M.; Capolino, F. Opt. Mater. Express 2011, 1, 1077-1089. doi:10.1364/OME.1.001077

29. Noginov, M. A.; Zhu, G.; Bahoura, M.; Adegoke, J.; Small, C. E.; Ritzo, B. A.; Drachev, V. P.; Shalaev, V. M. Opt. Lett. 2006, 31, 3022-3024. doi:10.1364/OL.31.003022

30. Strangi, G.; De Luca, A.; Ravaine, S.; Ferrie, M.; Bartolino, R. Appl. Phys. Lett. 2011, 98, 251912. doi:10.1063/1.3599566

31. Bergman, D. J.; Stockman, M. I. Phys. Rev. Lett. 2003, 90, 027402. doi:10.1103/PhysRevLett.90.027402

32. Lawandy, N. M. Appl. Phys. Lett. 2004, 85, 5040-5042. doi:10.1063/1.1825058

33. Protsenko, I. E.; Uskov, A. V.; Zaimidoroga, O. A.; Samoilov, V. N.; O'Reilly, E. P. Phys. Rev. A 2005, 71, 063812.

doi:10.1103/PhysRevA.71.063812 
34. Gordon, J. A.; Ziolkowski, R. W. Opt. Express 2007, 15, 2622-2653. doi:10.1364/OE.15.002622

35. Zheludev, N. I.; Prosvirnin, S. L.; Papasimakis, N.; Fedotov, V. A. Nat. Photonics 2008, 2, 351-354. doi:10.1038/nphoton.2008.82

36. Stockman, M. I. J. Opt. (Bristol, U. K.) 2010, 12, 024004. doi:10.1088/2040-8978/12/2/024004

37. Stockman, M. I. Phys. Rev. Lett. 2007, 98, 177404. doi:10.1103/PhysRevLett.98.177404

38. Mackay, T. G.; Lakhtakia, A. Phys. Rev. Lett. 2007, 99, 189701. doi:10.1103/PhysRevLett.99.189701

39. Kinsler, P.; McCall, M. W. Phys. Rev. Lett. 2008, 101, 167401. doi:10.1103/PhysRevLett.101.167401

40. Wuestner, S.; Pusch, A.; Tsakmakidis, K. L.; Hamm, J. M.; Hess, O. Phys. Rev. Lett. 2010, 105, 127401. doi:10.1103/PhysRevLett.105.127401

41. Stockman, M. I. Phys. Rev. Lett. 2011, 106, 156802. doi:10.1103/PhysRevLett.106.156802

42. Hill, M. T.; Oei, Y.-S.; Smalbrugge, B.; Zhu, Y.; de Vries, T.; van Veldhoven, P. J.; van Otten, F. W. M.; Eijkemans, T. J.; Turkiewicz, J. P.; de Waardt, H.; Geluk, E. J.; Kwon, S.-H.; Lee, Y.-H.; Nötzel, R.; Smit, M. K. Nat. Photonics 2007, 1, 589-594. doi:10.1038/nphoton.2007.171

43. Nezhad, M. P.; Simic, A.; Bondarenko, O.; Slutsky, B.; Mizrahi, A.; Feng, L.; Lomakin, V.; Fainman, Y. Nat. Photonics 2010, 4, 395-399. doi:10.1038/nphoton.2010.88

44. Lee, J. H.; Khajavikhan, M.; Simic, A.; Gu, Q.; Bondarenko, O.; Slutsky, B.; Nezhad, M. P.; Fainman, Y. Opt. Express 2011, 19, 21524-21531. doi:10.1364/OE.19.021524

45. Ding, K.; Liu, Z. C.; Yin, L. J.; Hill, M. T.; Marell, M. J. H.; van Veldhoven, P. J.; Nöetzel, R.; Ning, C. Z. Phys. Rev. B 2012, 85, 041301. doi:10.1103/PhysRevB.85.041301

46. Kwon, S.-H.; Kang, J.-H.; Seassal, C.; Kim, S.-K.; Regreny, P.; Lee, Y.-H.; Lieber, C. M.; Park, H.-G. Nano Lett. 2010, 10, 3679-3683. doi:10.1021/nl1021706

47. Oulton, R. F.; Sorger, V. J.; Zentgraf, T.; Ma, R.-M.; Gladden, C.; Dai, L.; Bartal, G.; Zhang, X. Nature 2009, 461, 629-632. doi:10.1038/nature08364

48. Lu, Y.-J.; Kim, J.; Chen, H.-Y.; Wu, C.; Dabidian, N.; Sanders, C. E.; Wang, C.-Y.; Lu, M.-Y.; Li, B.-H.; Qiu, X.; Chang, W.-H.; Chen, L.-J.; Shvets, G.; Shih, C.-K.; Gwo, S. Science 2012, 337, 450-453. doi:10.1126/science.1223504

49. Noginov, M. A.; Zhu, G.; Belgrave, A. M.; Bakker, R.; Shalaev, V. M.; Narimanov, E. E.; Stout, S.; Herz, E.; Suteewong, T.; Wiesner, U. Nature 2009, 460, 1110-1112. doi:10.1038/nature08318

50. Pan, J.; Chen, Z.; Chen, J.; Zhan, P.; Tang, C. J.; Wang, Z. L. Opt. Lett. 2012, 37, 1181-1183. doi:10.1364/OL.37.001181

51. Meng, X.; Guler, U.; Kildishev, A. V.; Fujita, K.; Tanaka, K.; Shalaev, V. M. Sci. Rep. 2013, 3, No. 1241. doi:10.1038/srep01241

52. Tribelsky, M. I.; Luk'yanchuk, B. S. Phys. Rev. Lett. 2006, 97, 263902. doi:10.1103/PhysRevLett.97.263902

53. Fan, X.; Shen, Z.; Luk'yanchuk, B. Opt. Express 2010, 18, 24868-24880. doi:10.1364/OE.18.024868

54. Johnson, P. B.; Christy, R. W. Phys. Rev. B 1972, 6, 4370-4379. doi:10.1103/PhysRevB.6.4370

55. Strickler, S. J.; Berg, R. A. J. Chem. Phys. 1962, 37, 814-822. doi:10.1063/1.1733166

56. Khurgin, J. B.; Sun, G. Opt. Express 2012, 20, 15309-15325. doi:10.1364/OE.20.015309

57. Prodan, E.; Radloff, C.; Halas, N. J.; Nordlander, P. Science 2003, 302, 419-422. doi:10.1126/science. 1089171
58. Baranov, D. G.; Andrianov, E. S.; Vinogradov, A. P.; Lisyansky, A. A. Opt. Express 2013, 21, 10779-10791. doi:10.1364/OE.21.010779

59. Vial, A.; Laroche, T. Appl. Phys. B 2008, 93, 139-143. doi:10.1007/s00340-008-3202-4

60. Prokopeva, L. J.; Trieschmann, J.; Klar, T. A.; Kildishev, A. V. Proc. SPIE 2011, 8172, 81720B. doi:10.1117/12.898619

61. Garcia de Abajo, F. J. J. Phys. Chem. C 2008, 112, 17983-17987. doi:10.1021/jp807345h

62. Zuloaga, J.; Prodan, E.; Nordlander, P. Nano Lett. 2009, 9, 887-891. doi:10.1021/nl803811g

63. Hoffmann, J.; Hafner, C.; Leidenberger, P.; Hesselbarth, J.; Burger, S. Proc. SPIE 2009, 7390, 73900J. doi:10.1117/12.828036

\section{License and Terms}

This is an Open Access article under the terms of the Creative Commons Attribution License

(http://creativecommons.org/licenses/by/2.0), which permits unrestricted use, distribution, and reproduction in any medium, provided the original work is properly cited.

The license is subject to the Beilstein Journal of

Nanotechnology terms and conditions:

(http://www.beilstein-journals.org/bjnano)

The definitive version of this article is the electronic one which can be found at: doi:10.3762/bjnano.4.110 